\title{
CARACTERÍsticas MoRfofisiológicas de Biótipos de Euphorbia heterophylla com ResistênCIA A Diferentes MECANISMOS DE AÇÃo HERBICIDA $^{1}$
}

\author{
Morphophysiological Characteristics of Euphorbia heterophylla Biotypes Resistant to Different \\ Herbicide Action Mechanisms
}

\author{
TREZZI, M.M. ${ }^{2}$, PORTES, E.D.S. ${ }^{3}$, SILVA, H.L. ${ }^{3}$, GUSTMAN, M.S. ${ }^{4}$, DA SILVA, R.P. ${ }^{4}$ e \\ FRANCHIN, E. ${ }^{5}$
}

\begin{abstract}
RESUMO - A resistência de plantas daninhas aos herbicidas é um dos maiores entraves da agricultura mundial. No Brasil, Euphorbia heterophylla (EPHHL) obtém destaque devido ao fato de apresentar resistência a mais de um mecanismo de ação de herbicidas. O presente trabalho foi conduzido com o objetivo de identificar características morfofisiológicas e a adaptabilidade ecológica entre biótipos de Euphorbia heterophylla com resistência múltipla a inibidores da ALS e Protox e com resistência apenas a inibidores da ALS e suscetivel, oriundos da região sudoeste do Paraná. O experimento foi desenvolvido em casa de vegetação. O delineamento utilizado foi o completamente casualizado, com quatro repetições. As características morfofisiológicas avaliadas foram: matéria seca de folhas+cotilédones, de raizes, de caule+pecíolos e total, estatura, número de ramificações por planta, área foliar e número de folhas por planta. Nas determinações efetuadas mais tardiamente, plantas do biótipo S apresentaram menores matérias secas de folhas+cotilédones, caules+pecíolos e total. Plantas do biótipo $\mathrm{S}$ também apresentaram menores área foliar, número de ramificações e estatura em avaliações mais tardias, comparativamente aos biótipos $R$ a ALS e R a Protox.
\end{abstract}

Palavras-chave: adaptabilidade, competição, comportamento biológico, estatura, leiteira.

\begin{abstract}
Weed resistance to herbicides is one of the major barriers to agriculture worldwide. In Brazil, Euphorbia heterophylla (EPHHL) is especially known for presenting resistance to more than one herbicide action mechanism. This work was carried out to identify morphophysiological characteristics and ecological adaptability between Euphorbia heterophylla biotypes with multiple resistance to ALS inhibitors and PROTOX, and with resistance only to ALS inhibitors and susceptible from Southwestern Paraná. The experiment was conducted in a greenhouse and arranged in a completely randomized design, with four replications. The morphophysiological characteristics evaluated were: dry matter of leaves + cotyledons, roots, stems+petioles and total, plant height, number of branches per plant, leaf area and number of leaves per plant. In determinations made at a later time, biotype S presented smaller dry matter of leaves+cotyledons, stems+petioles and total, as well as smaller leaf area, number of leaves per plant and height than biotypes $R$ to $A L S$ and $R$ to Protox.
\end{abstract}

Keywords: adaptability, competition, biological behavior, height, wild poinsettia

1 Recebido para publicação em 27.5.2009 e na forma revisada em 11.12.2009.

2 Professor Adjunto, Dr., Universidade Tecnológica Federal do Paraná - UTFPR, Via do Conhecimento, Km 01, Caixa Postal

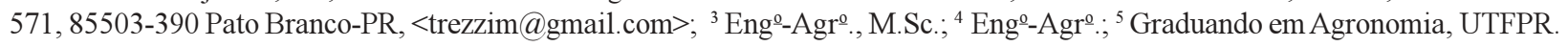




\section{INTRODUÇÃO}

Atualmente, a resistência de plantas daninhas a herbicidas é um dos principais problemas enfrentados pelos diferentes segmentos envolvidos na agricultura. Entre as principais consequências da resistência de plantas daninhas a herbicidas estão a limitação ou inviabilização do uso de determinados herbicidas, perda de áreas para semeadura, perda de rendimento e qualidade dos produtos das culturas agrícolas, necessidade de reaplicação de herbicidas e mudanças em sistemas de produção. Em muitos casos, baixos níveis de resistência (Vidal et al., 2007a) determinam a elevação das doses de herbicidas pelos agricultores, resultando em maior impacto ambiental e elevação dos custos de produção, com consequente redução da competitividade na comercialização do produto final.

Até hoje, no Brasil, foram identificados biótipos resistentes (R) a herbicidas em 18 espécies de plantas daninhas (Heap, 2009). Entre elas, Euphorbia heterophylla (EPHHL) é a que apresenta resistência ao maior número de mecanismos de ação herbicida: inibidores da acetolactato sintase (ALS) (Gazziero et al., 1998; Vidal \& Merotto Jr., 1999), múltipla a inibidores da ALS e protoporfirinogênio oxidase (Protox) (Trezzi et al., 2005; Trezzi et al., 2006) e múltipla a inibidores da ALS e EPSPs (Vidal et al., 2007b).

Em 2004, em áreas cultivadas com soja localizadas na região sudoeste do Paraná, foram identificados dois biótipos de EPHHL que não respondiam à aplicação das doses recomendadas dos herbicidas imazethapyr (inibidor da ALS) e fomesafen (inibidor da Protox) e que apresentavam resistência cruzada a outros herbicidas inibidores da ALS e da Protox (Trezzi et al., 2005). A investigação do mecanismo de resistência em um desses biótipos indicou, preliminarmente, que a metabolização não é o mecanismo gerador da resistência (Nunes et al., 2007). Níveis de controle inferiores no biótipo R em comparação ao suscetível (S), com aplicação de fomesafen apenas na parte aérea das plantas, e diferenças de controle significativamente menores entre os biótipos, quando este herbicida foi aplicado no solo, indicaram que o mecanismo de resistência está relacionado à absorção inferior dos inibidores da Protox pela parte aérea das plantas $\mathrm{R}$ (Trezzi et al., 2009).

A probabilidade de infestação de uma área com indivíduos $\mathrm{R}$ depende de vários fatores, como adaptabilidade ecológica e prolificidade do indivíduo, longevidade e dormência das sementes da espécie ou biótipo sob seleção, frequência de utilização de herbicidas de um único mecanismo de ação e sua persistência, eficácia do herbicida e métodos adicionais empregados no controle de plantas daninhas (Gressel \& Segel, 1990; Maxwell et al., 1990; Putwain \& Mortimer, 1989). A capacidade de sobrevivência e reprodução de um biótipo em uma população determina a sua adaptabilidade ecológica, a qual depende de características biológicas, como taxas de germinação, crescimento e produção de sementes (Christoffoleti, 2001). Para que medidas de prevenção e manejo possam ser recomendadas de forma racional e com base em informações consistentes de previsão da evolução da resistência em populações de plantas daninhas, é necessário que o comportamento biológico dos biótipos S e R seja caracterizado (Gill et al., 1996).

A investigação da adaptabilidade ecológica tem sido feita em vários biótipos de espécies resistentes a inibidores de ALS e, até hoje, não se demonstrou, em geral, desempenho inferior de biótipos R (Thompson et al., 1994; O'Donovan et al., 1994; Christoffoleti et al., 1997; Christoffoleti, 2001). No Brasil, características morfofisiológicas e adaptabilidade ecológica de biótipos de EPHHL resistentes a inibidores da ALS foram comparadas com o biótipo S e não se observou prejuízo ao desenvolvimento do biótipo R (Vidal \& Trezzi, 1999; Trezzi \& Vidal, 2000; Brighenti et al., 2001). No entanto, ainda é desconhecido o comportamento dos biótipos de EPHHL com resistência simultânea a inibidores da ALS e Protox, em relação a biótipos suscetiveis e resistentes a inibidores da ALS.

O presente trabalho foi realizado com o objetivo de comparar características morfofisiológicas e a adaptabilidade ecológica entre biótipos de EPHHL com resistência múltipla a inibidores da ALS e Protox, com resistência apenas a inibidores da ALS e suscetivel, oriundos da região sudoeste do Paraná. 


\section{MATERIAL E MÉTODOS}

Foi conduzido experimento em vasos com capacidade para $4.000 \mathrm{~cm}^{3}$, em casa de vegetação do Curso de Agronomia da UTFPR, em delineamento completamente casualizado, com quatro repetições, no período de outubro a dezembro de 2006. Os tratamentos foram arranjados em um fatorial $3 \times 8$, em que o primeiro fator representou os biótipos de EPHHL: suscetivel (S), com resistência a inibidores da ALS (R a ALS) e com resistência simultânea a inibidores da ALS e da Protox (R a Protox); o segundo fator foi constituído por sete épocas de determinação de características morfofisiológicas das plantas de EPHHL $(21,28,35,42,49,56$ e 63 dias após a emergência das plantas (DAE)).

As sementes dos biótipos R a ALS, R a Protox e $\mathrm{S}$ foram coletadas, respectivamente, em áreas de lavoura de soja pertencentes aos municípios de Pato Branco, Vitorino e Renascença, todos localizados na região sudoeste do Paraná. A confirmação dos biótipos com resistência a inibidores da ALS e resistência múltipla a inibidores da Protox e ALS foi documentada, respectivamente, por Trezzi et al. (2005) e Trezzi et al. (2006). Foram depositadas em torno de 15 sementes por vaso. Após a emergência, realizou-se o desbaste das plantas, deixando-se três em cada vaso. Os vasos foram preenchidos com solo coletado na Área Experimental Agronômica da UTFPR, localizada em Pato Branco (PR). O solo é classificado, segundo o Sistema Brasileiro de Classificação de Solos, como Latossolo Vermelho distrófico típico, contendo 65\% de argila (Embrapa, 2006). Os vasos foram irrigados visando à manutenção do solo em capacidade de campo.

As seguintes determinações foram realizadas a cada semana: estatura, área foliar, número de folhas, número de ramificações, matéria seca de cotilédones+folhas, de caules+pecíolos e das raízes. A área de cotilédones+folhas foi medida através de integrador de área foliar, e a estatura das plantas, por meio de régua graduada. A matéria seca foi obtida pela secagem em estufa a $65{ }^{\circ} \mathrm{C}$ das partes da planta, até se obter peso constante. Foi determinado também o momento de início de surgimento das estruturas reprodutivas nos biótipos. Realizou-se a análise de variância pelo teste $\mathrm{F}$, e as médias dos biótipos foram comparadas pelo teste de Tukey a 5\% de probabilidade de erro.

\section{RESULTADOS E DISCUSSÃO}

$\mathrm{O}$ início do florescimento dos biótipos $\mathrm{R} \mathrm{a}$ Protox e $\mathrm{S}$ ocorreu a partir da avaliação aos $56 \mathrm{DAE}$, enquanto para o biótipo R a ALS ele não ocorreu até o encerramento do experimento. Esse desenvolvimento reprodutivo mais tardio do biótipo R a ALS foi confirmado em avaliações posteriores, não documentadas no presente artigo. O desenvolvimento reprodutivo mais tardio do biótipo R a ALS poderia proporcionar menor número de gerações de sementes e, consequentemente, menor número de sementes no banco, o que pode determinar menor probabilidade de seleção de indivíduos desse biótipo, comparada à dos demais (JASIENIUK et al., 1996).

Os biótipos de EPHHL apresentaram aumento na produção de matéria seca de cotilédones+folhas até o período de $56 \mathrm{DAE}$. No último período de avaliação, aos $63 \mathrm{DAE}$, as matérias secas de cotilédones+folhas dos biótipos $\mathrm{R}$ a Protox e $\mathrm{S}$ foram reduzidas em relação a 56 DAE (Figura 1). Os biótipos de EPHHL apresentaram diferença significativa entre as médias de matéria seca de folhas+cotilédones somente aos $63 \mathrm{DAE}$ (Figura 1), em que o biótipo R a ALS $(0,982 \mathrm{~g}$ por planta) superou significativamente o biótipo suscetivel $(0,684 \mathrm{~g}$ por planta), porém não diferiu significativamete do biótipo $R$ a Protox $(0,829$ $\mathrm{g}$ por planta). As reduções das matérias secas de cotilédones+folhas dos biótipos $\mathrm{R}$ a Protox $\mathrm{e}$ $\mathrm{S}$ podem ser explicadas pelo balanço negativo entre acréscimo de folhas novas e queda de folhas baixeiras, decorrente da senescência. Mesmo em caso de balanço foliar não negativo, é possivel que as folhas mais novas apresentem menor área foliar e matéria seca do que as que foram perdidas.

Até os $56 \mathrm{DAE}$, todos os biótipos apresentaram área foliar crescente, não diferindo entre si (Figura 2). O número de folhas das plantas de EPHHL somente diferiu significativamente entre os biótipos no período de avaliação aos $56 \mathrm{DAE}$ (Figura 2), em que o biótipo $\mathrm{R}$ a Protox apresentou número de folhas superior significativamente ao dos biótipos 


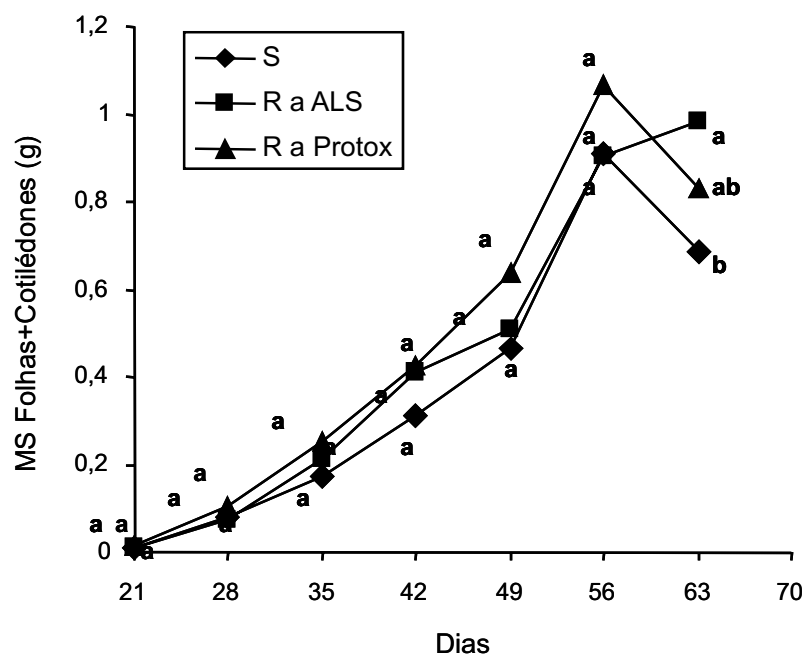

Figura 1 - Matéria seca de folhas e cotilédones (g) de planta dos biótipos resistentes a inibidores da ALS (R a ALS), a inibidores da Protox (R a Protox) e suscetível (S) de EPHHL, determinada em sete períodos distintos.

$\mathrm{R}$ a ALS e S. O biótipo R a Protox apresentou balanço foliar negativo (Figura 2) aos 63 DAE. Embora não tenha apresentado balanço foliar negativo (Figura 2), o biótipo $\mathrm{S}$ mostrou decréscimo da área foliar aos $63 \mathrm{DAE}$, em comparação aos outros dois (Figura 3). Esse comportamento pode explicar o decréscimo da matéria seca de folhas + cotilédones na última avaliação para os biótipos com resistência a Protox e suscetivel.

Em experimento realizado em Porto Alegre (RS) em estação estival, comparando três biótipos R a ALS com um S, com coletas realizadas a cada dez dias, totalizando cinco coletas, Trezzi \& Vidal (2000) constataram que no biótipo $\mathrm{S}$ e em um R, proveniente de Passo Fundo, houve estabilização da área foliar a partir dos 43 dias após a emergência, enquanto para os biótipos provenientes de Rio Pardo e Não-Me-Toque a área foliar continuou crescendo até o final do experimento, aos 53 DAE. Em Londrina (PR), em que foram realizadas 13 coletas com intervalos de 14 dias, Brighenti et al. (2001) determinaram as máximas área foliar e produção de matéria seca de folhas, respectivamente, aos 113 e 99 dias após a semeadura. Características dos biótipos e de fatores abióticos, como a soma térmica e o fotoperíodo, podem explicar as diferenças entre os locais estudados. Diferenças entre os

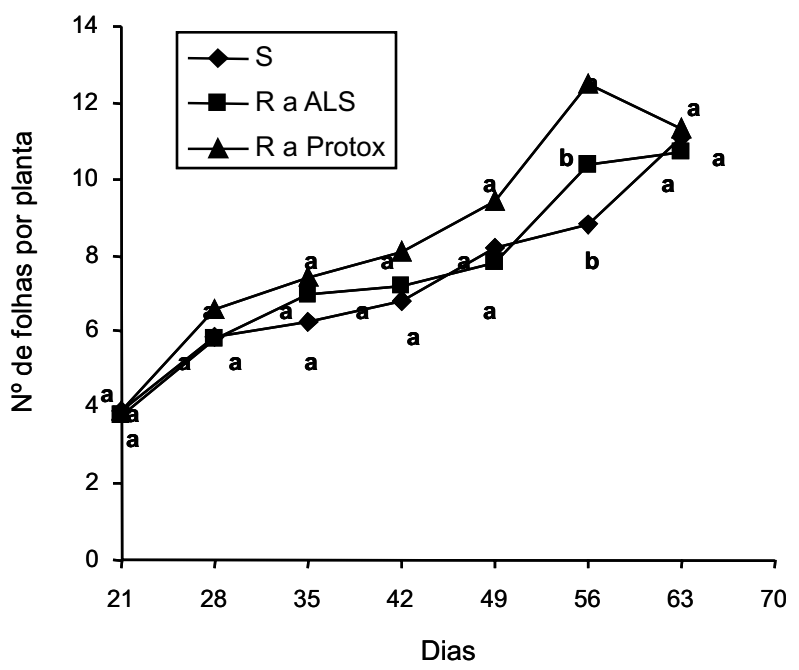

Figura 2 - Número de folhas por planta dos biótipos resistentes a inibidores da ALS (R a ALS), a inibidores da Protox (R a Protox) e suscetível (S) de EPHHL, determinado em sete períodos distintos.

biótipos estudados indicam a existência de variabilidade genética entre eles, já constatada em estudos com EPHHL (Vasconcelos et al., 2000), o que permite seleção de características adaptativas, associadas principalmente à maior sobrevivência a práticas de manejo e ao momento em que a colheita das lavouras é efetuada.

Quanto à produção de matéria seca de caules+pecíolos (Figura 4), o biótipo R a Protox diferiu significativamente dos biótipos R a ALS e suscetível nos períodos de 49 e de 56 DAE. Aos 63 DAE, os biótipos R a Protox e R a ALS não diferiram entre si, mas diferiram significativamente do biótipo $\mathrm{S}$, o qual foi inferior em produção de matéria seca de caules+pecíolos.

Houve comportamento similar entre os biótipos na maior parte do tempo quanto às médias de matéria seca de raízes. No entanto, aos 56 DAE, o biótipo $R$ a Protox $(1,139 \mathrm{~g})$ superou o R a ALS (0,736 g) e $\mathrm{S}(0,695 \mathrm{~g})$ (Figura 5). Houve recuperação do desenvolvimento de raízes do biótipo com resistência a ALS na última avaliação, não havendo diferença significativa entre as médias de matéria seca de raízes nessa avaliação.

Na análise das Figuras 1, 4 e 5, que comparam a acumulação de matéria seca ao longo do ciclo dos biótipos, percebe-se nitidamente 


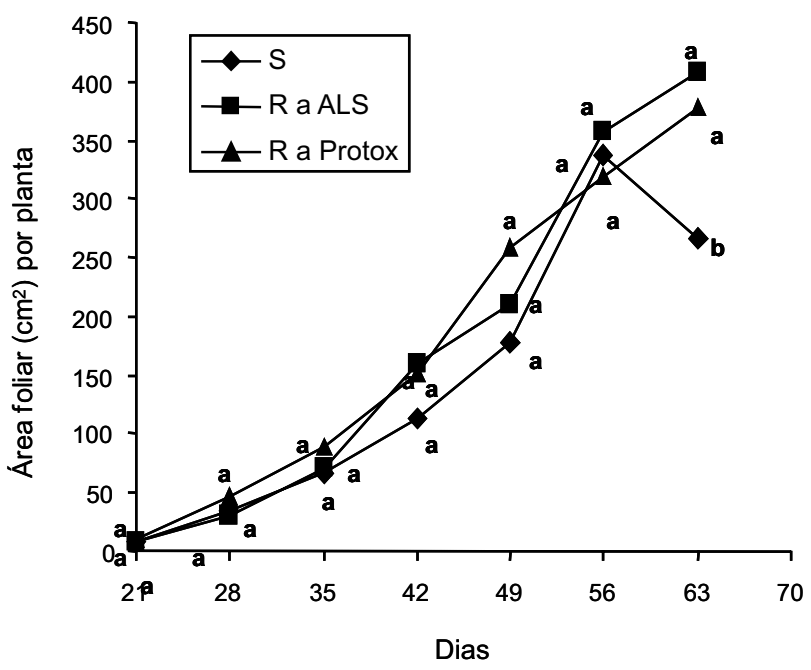

Figura 3 - Área foliar $\left(\mathrm{cm}^{2}\right)$ por planta dos biótipos resistentes a inibidores da ALS (R a ALS), a inibidores da Protox (R a Protox) e suscetível (S) de EPHHL, determinada em sete períodos distintos.

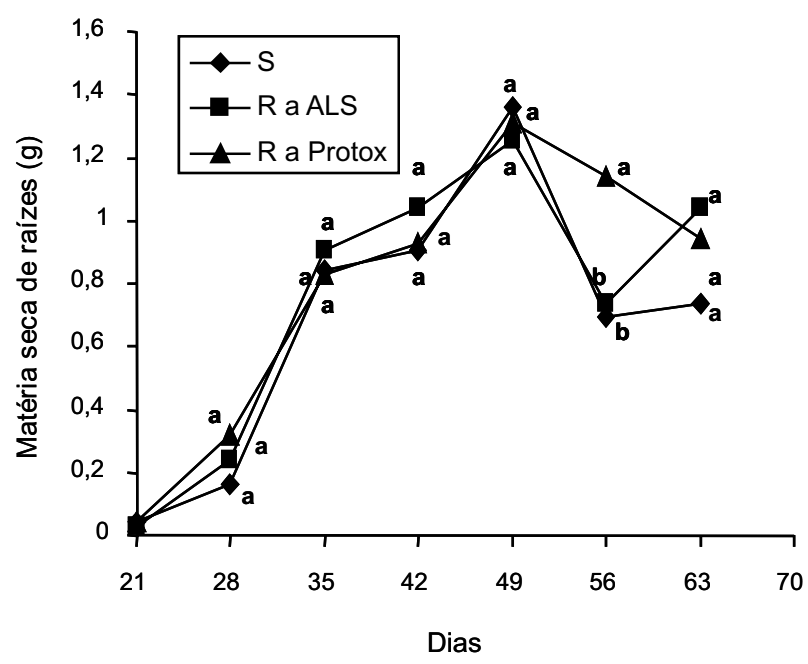

Figura 5 - Matéria seca de raízes (g por planta) dos biótipos resistentes a inibidores da ALS (R a ALS), a inibidores da Protox (R a Protox) e suscetível (S) de EPHHL, determinada em sete períodos distintos.

tendência de que as plantas invistam, até os 49 DAE, em maior quantidade de recursos no desenvolvimento de raízes, seguido do desenvolvimento de folhas, com baixo investimento de recursos em caules+pecíolos. A partir de $49 \mathrm{DAE}$, os recursos passaram a ser investidos principalmente em caules+peciolos. Esse fato indica priorização do investimento inicial das

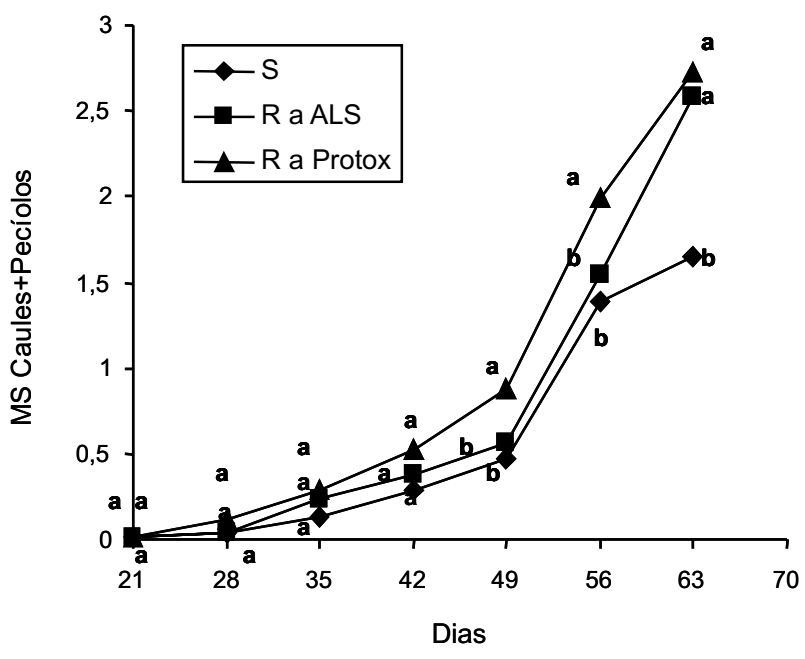

Figura 4 - Matéria seca de caules e pecíolos (g) de planta dos biótipos resistentes a inibidores da ALS (R a ALS), a inibidores da Protox (R a Protox) e suscetível (S) de EPHHL, determinada em sete períodos distintos.

plantas em estruturas de assimilação de água, nutrientes e $\mathrm{CO}_{2}$, para posteriormente destinar fotoassimilados para estruturas de sustentação das plantas e estruturas reprodutivas. Esse tipo de alocação estratégica de recursos já havia sido detectado em outro experimento, implantado em estação estival, comparando biótipos de EPHHL com R a ALS e S (Trezzi \& Vidal, 2000). No entanto, comparando os mesmos biótipos em época de semeadura de outono, as plantas priorizaram o desenvolvimento de folhas e caules em estádios de desenvolvimento inicial (Vidal \& Trezzi, 1999), ou seja, a partição de recursos entre partes das plantas de EPHHL é influenciada por fatores do ambiente - nesse caso, notadamente a temperatura.

As plantas dos biótipos R a ALS e R a Protox iniciaram a emissão de ramificações a partir de $35 \mathrm{DAE}$, enquanto a ramificação das plantas do biótipo $\mathrm{S}$ só ocorreu a partir da última determinação, aos 63 DAE (Figura 6). O número de ramificações emitidas pelo biótipo $\mathrm{R}$ a ALS chegou a um máximo de aproximadamente dois por planta, superior ao dos biótipos R a Protox e ao S. Essa característica é importante, pois confere maior plasticidade às plantas (Radosevich et al., 1997), o que pode significar ocupação maior dos espaços pelas plantas desse biótipo, especialmente em 
situações em que elas estejam em menor densidade e em que a capacidade de suporte do ambiente é maior. Esse atributo pode aumentar a capacidade competitiva desse biótipo com espécies cultivadas e também entre biótipos de EPHHL que estejam na mesma área. No entanto, a habilidade competitiva deve ser analisada considerando-se em conjunto outras características do biótipo e, preferencialmente, em experimentos de competição entre biótipos. Também, a capacidade superior de ramificação pode ser determinante da produção de sementes no final do ciclo, já que a lei do rendimento final constante determina que a produção de sementes de uma espécie produzida por área tende a ser constante (Radosevich et al., 1997), desde que haja uma densidade mínima e capacidade plástica das plantas.

A matéria seca total acumulada no período experimental não diferiu significativamente entre os biótipos de EPHHL no período entre 21 e 49 DAE (Figura 7). No entanto, 56 DAE, a produção de matéria seca total do biótipo $\mathrm{R}$ a Protox apresentou diferença significativa em relação aos biótipos R a ALS e S. No último período de avaliação, aos $63 \mathrm{DAE}$, os biótipos $\mathrm{R}$ a Protox e R a ALS apresentaram praticamente a mesma produção de matéria seca total, diferindo significativamente e sendo superior à produção de matéria seca total do biótipo $\mathrm{S}$.
A superioridade do biótipo $\mathrm{R}$ a Protox aos 56 DAE foi devida, basicamente, ao maior desenvolvimento de raízes nesse período (Figura 5). Contribuíram para a menor matéria seca total em $\mathrm{S}$ no final do ciclo, em relação a $\mathrm{R}$ a ALS e $\mathrm{R}$ a Protox, as menores matérias secas de folhas, caules e pecíolos no período final de avaliação (Figuras 1 e 4).

O comportamento da estatura ao longo das avaliações foi similar ao da matéria seca total, porém as diferenças entre biótipos ocorreram em períodos diferentes. A Figura 8 mostra que os biótipos de EPHHL apresentaram diferença significativa de estatura somente aos 42 e 63 DAE. Aos 42 DAE, o biótipo R a Protox apresentou estatura significativamente superior à dos biótipos R a ALS e S. Aos $63 \mathrm{DAE}$, o biótipo $\mathrm{R}$ a Protox apresentou estatura superior, mas não diferiu significativamente do biótipo $R$ a ALS, ambos possuindo estaturas superiores à do S. Essa característica é importante porque influencia a capacidade competitiva por radiação solar. Segundo Callaway (1992), plantas que apresentam elevadas estatura e área foliar geralmente correspondem àquelas que possuem habilidade competitiva superior.

Conclui-se que existem diferenças expressivas entre os biótipos de EPHHL quanto às variáveis avaliadas. As diferenças mais expressivas foram encontradas entre o grupo

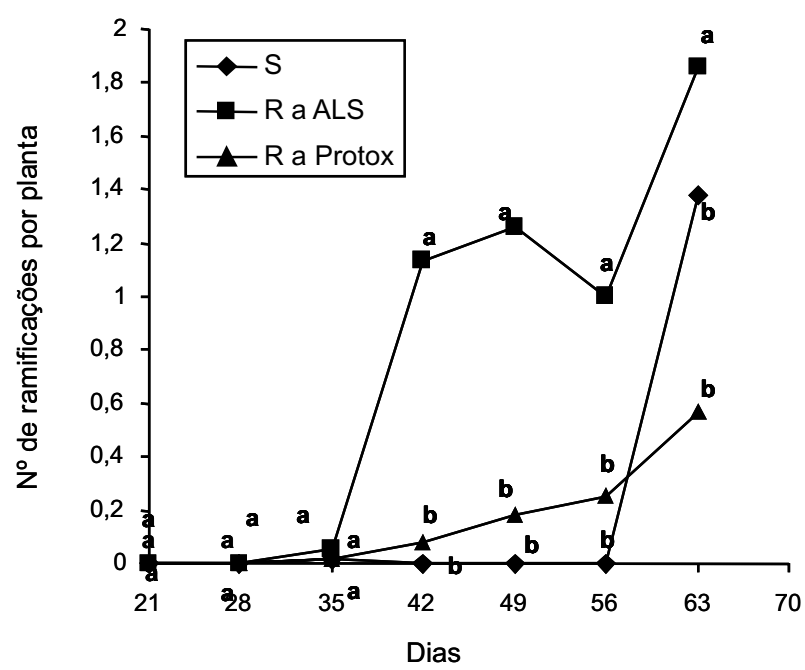

Figura 6 - Número de ramificações por planta dos biótipos resistentes a inibidores da ALS (R a ALS), a inibidores da Protox (R a Protox) e suscetível (S) de EPHHL, determinado em sete períodos distintos

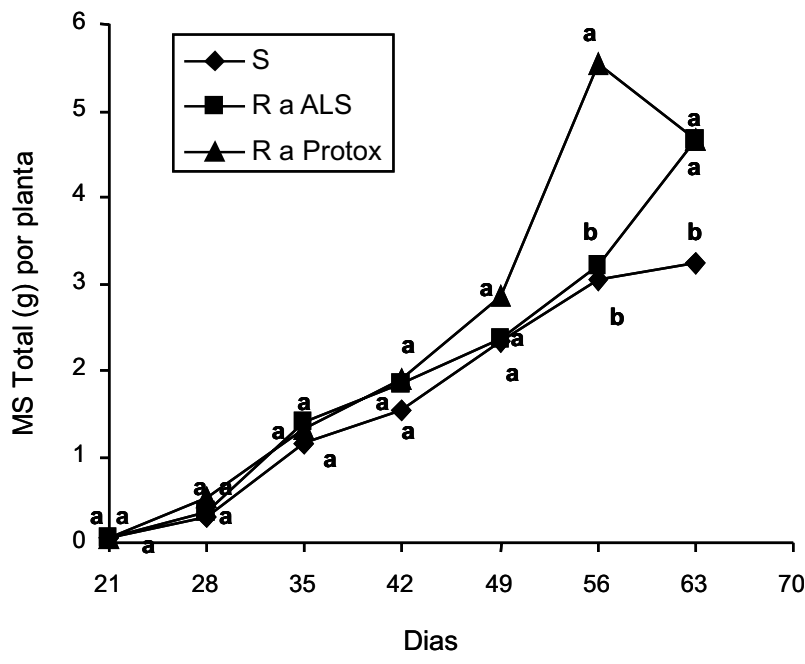

Figura 7 - Matéria seca total (g) de planta dos biótipos resistentes a inibidores da ALS (R a ALS), a inibidores da Protox (R a Protox) e suscetível (S) de EPHHL, determinado em sete períodos distintos. 


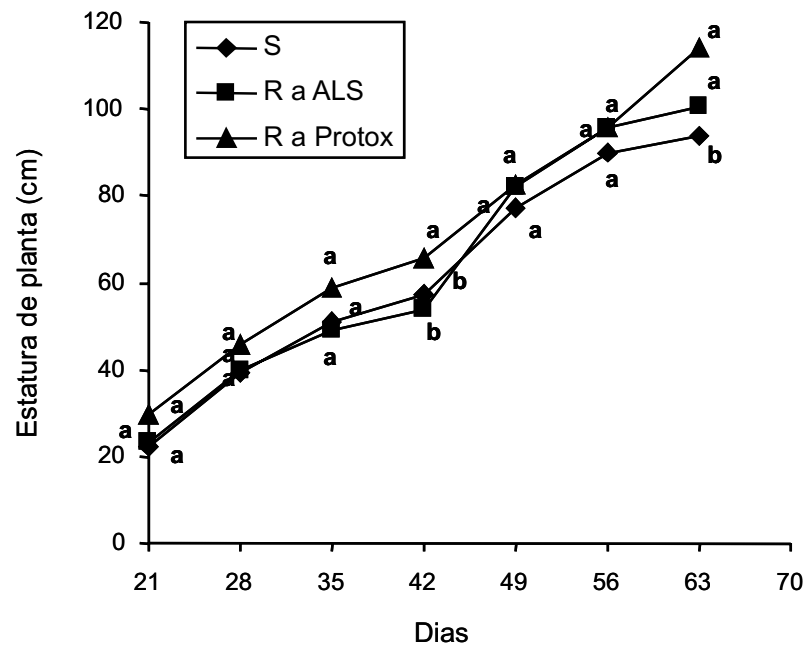

Figura 8 - Estatura $(\mathrm{cm})$ de planta dos biótipos resistentes a inibidores daALS (R aALS), a inibidores da Protox (R Protox) e suscetível (S) de EPHHL, determinada em sete períodos distintos

dos biótipos $\mathrm{R}(\mathrm{R}$ a inibidores da ALS e a inibidores da Protox) e o biótipo S. Nas determinações efetuadas mais tardiamente, o biótipo $\mathrm{S}$ originário do município de Renascença (PR) apresentou menores matérias secas de folhas+cotilédones, caules+pecíolos e total. Suas plantas também mostraram menores área foliar, número de ramificações e estatura em avaliações mais tardias, comparativamente aos biótipos R a ALS e R a Protox.

Segundo Ogg \& Seefeldt (1999), plantas com rápido incremento em estatura geralmente levam vantagem competitiva sobre as concorrentes, posteriormente. Para Blackshaw (1994), a rapidez de tomada do espaço aéreo constitui-se em importante característica para o sucesso competitivo. No entanto, algumas características depreciativas do biótipo $\mathrm{S}$ somente foram detectadas a partir de estádios mais avançados das plantas, e não se sabe qual exatamente o seu impacto sobre a adaptabilidade em relação a outros biótipos de EPHHL.

$\mathrm{O}$ biótipo R a ALS atingiu mais lentamente o estádio reprodutivo, porém apresentou capacidade de emissão de ramificações mais acentuada do que os demais biótipos avaliados. Se por um lado o ciclo mais longo pode significar prejuízo para o número de gerações de produção de sementes, a maior ramificação pode representar maior capacidade plástica determinante de maior produção de sementes em uma geração - importante para alimentar o banco de sementes e determinar a probabilidade de seleção nas próximas gerações. Em que pesem as diferenças morfo-fisiológicas entre os biótipos testados, a existência ou não de diferenças de adaptabilidade ecológica entre eles poderia ser mais bem definida em experimentos em que eles fossem colocados em competição entre si.

São desconhecidos, até o momento, resultados de experimentos avaliando características morfofisiológicas de biótipos de espécies resistentes a inibidores da Protox. Investigação da adaptabilidade ecológica tem sido feita em vários biótipos de espécies resistentes a inibidores de ALS, e até hoje não se demonstrou, em geral, desempenho inferior de biótipos resistentes (Thompson et al., 1994; Christoffoleti et al., 1997; Vidal \& Trezzi, 2000; Christoffoleti, 2001; Park et al., 2004), com exceção de um biótipo de Amaranthus blitoides (Sibony \& Rubin, 2003) com resistência múltipla a inibidores da ALS e do fotossistema II (FS II), em que a penalidade adaptativa esteve associada ao mecanismo de resistência a inibidores do FSII. Na maioria dos casos em que houve diferenças morfofisiológicas entre biótipo suscetivel e resistente a inibidores da ALS em experimentos sem competição, posteriormente, essas diferenças não foram confirmadas em condições competitivas (Alcocer-Ruthling et al., 1992; O’Donovan et al., 1994).

Os resultados do presente experimento não demonstraram qualquer vantagem competitiva do biótipo $\mathrm{S}$ em relação aos biótipos $\mathrm{R}$ a ALS e R a Protox. Portanto, a adoção de estratégias preventivas é importante, pois a supressão da aplicação de herbicidas selecionadores das populações resistentes não resultará, em princípio, em aumento da frequência de indivíduos suscetiveis nas áreas.

\section{LITERATURA CITADA}

BLACKSHAW, R. E. Differential competitive ability of winter wheat cultivars against downy brome. Agron. J., v. 86, n. 4, p. 649-654, 1994.

BRIGHENTI, A. M. et al. Análise do crescimento de biótipos de amendoim-bravo (Euphorbia heterophylla) resistente e suscetível aos herbicidas inibidores da ALS. Planta Daninha, v. 19, n. 1, p. $51-59,2001$. 
CALLAWAY, M. B. A compendium of crop varietal tolerance to weeds. Am. J. Alter. Agric., v. 7, n. 4, p. 169-180, 1992.

CHRISTOFOLETTI, P. J. Análise comparativa do crescimento de biótipos de picão preto (Bidens pilosa) resistente e suscetível aos herbicidas inibidores da ALS. Planta Daninha, v. 19 , n. 1, p. $75-83,2001$

CHRISTOFFOLETI, P. J.; WESTRA, P. ; MOORE, F. Growth analysis of sulfonylurea-resistant and susceptible kochia (Kochia scoparia). Weed Sci., v. 45, p. 691-695, 1997.

EMPRESA BRASILEIRA DE PESQUISAAGROPECUÁRIA - EMBRAPA. Centro Nacional de Pesquisa Agropecuária de Solos. Sistema brasileiro de classificação de solo. Rio de Janeiro: Embrapa Solos, 2006. 306 p.

GAZZIERO, D. L. P. et al. Resistência de amendoim-bravo aos herbicidas inibidores da ALS. Planta Daninha, v. 16, n. 2, p. $117-125,1998$.

GILL, G. S.; CONSENS, R. D.; ALLAN, M. R. Germination, growth, and development of herbicide resistant and susceptible populations of rigid ryegrass (Lolium rigidum). Weed Sci., v. 44 , p. 252-256, 1996

GRESSEL, J.; SEGEL, L. A. Modeling the effectiveness of herbicide rotations and mixtures as strategies to delay of preclude resistance. Weed Technol., v. 4, p. 186-198, 1990

HEAP, I. International survey of resistant weeds. Disponível em: $<$ http://www. weedsciencie.org/in.asp $>$. Acesso em: 15 maio de 2009

JASIENIUK, M.; BRÛLÉ-BABEL, A. L.; MORRISON, I. N. The evolution and genetics of herbicide resistance in weeds. Weed Sci., v. 44, p. 176-193, 1996.

MAXWELL, B. D.; ROUSH, M. L.; RADOSEVICH, J. R Predicting the evolution and dynamics of herbicide resistance in weed populations. Weed Technol., v. 4, p. 2-13, 1990

NUNES, A. L. et al. Metabolização diferencial de fomesafen e lactofen entre biótipos de Euphorbia heterophylla: causa da resistência a herbicidas inibidores da PROTOX? Syner. Scy. UTFPR, v. 2, p. 9, 2007

O'DONOVAN, J. T. et al. Investigation of a chlorsulfuronresistant chickweed [Stellaria media (L.) Vill.] population.

Can. J. Plant Sci., v. 74, p. 693-697, 1994

OGG JR., A. G.; SEEFELDT, S. S. Characterizing traits that enhance the competitiveness of winter wheat (Triticum aestivum) against jointed goatgrass (Aegilops cylindrica) Weed Sci., v. 47, n. 1, p. 74-80, 1999

PARK, K. W. et al. Ecological fitness of acetolactate synthase inhibitor-resistant and susceptible downy brome (Bromus tectorum) biotypes. Weed Sci., v. 52, p. 768-773, 2004

PUTWAIN, P. D.; MORTIMER, A. M. The resistance of weeds to herbicides: rational approaches for containment of a growing problem. Brighton Crop Protection Conference Weeds. p. 285-294, 1989.
RADOSEVICH, S.; HOLT, J.; GHERSA, C. Weed ecology: implications for management. New York: John Wiley \& Sons, 1997, 588 p.

SIBONY, M.; RUBIN, B. The ecological fitness of ALSresistant Amaranthus retroflexus and multiple-resistant Amaranthus blitoides. Weed Res., v. 43, p. 40-47, 2003.

THOMPSON, C. R.; THILL, D. C.; SHAFII, B. Growth and competitiveness of sulfonylurea-resistant and susceptible kochia (Kochia scoparia). Weed Sci., v. 42, p. 172-179, 1994.

TREZZI, M. M. et al. Multiple resistance of acetolactate synthase and protoporphyrinogen oxidase inhibitors in Euphorbia heterophylla biotypes. J. Environ. Sci. Health., Part B, v. B40, n. 1, p. 1-9, 2005

TREZZI, M. M. et al. Bioensaios para identificação de biótipos de Euphorbia heterophylla com resistência múltipla a inibidores da ALS e da PROTOX. Planta Daninha, v. 24, n. 3, p. $563-571,2006$.

TREZZI, M. M. et al. Resposta a imidazolinonas, sulfoniluréias e sulfonanilidas de quinze biótipos de Euphorbia heterophylla com suspeita de resistência a inibidores da ALS. In: CONGRESSO BRASILEIRO DA CIÊNCIA DAS PLANTAS DANINHAS, 25., 2006, Brasília (DF). Resumos... Brasília (DF): SBCPD/UNB/Embrapa Cerrados, 2006. p. 561-561

TREZZI, M. M. et al. Local de absorção de fomesafen como mecanismo de resistência em biótipo de Euphorbia heterophylla resistente aos inibidores da Protox. Planta Daninha, v. 27, p. 139-148, 2009.

TREZZI, M. M.; VIDAL, R. A. Crescimento estival de três biótipos de leiteira resistentes e um suscetível aos herbicidas inibidores da acetolactato sintase. R. Bras. Herbic., v. 1, n. 3, p. 255-260, 2000.

VASCONCELOS, M. J. V. et al. Variabilidade genética em biótipos de leiteiro de Londrina/PR. Planta Daninha, v. 18, n. 2 , p. $285-292,2000$

VIDAL, R. A.; MEROTTO JR., A. Resistência de amendoim bravo (Euphorbia heterophylla L.) aos herbicidas inibidores da enzima acetolactato sintase. Planta Daninha, v. 17, n. 3, p. $367-373,1999$.

VIDAL, R. A.; TREZZI, M. M. Desenvolvimento comparativo entre biótipos de leiteira (Euphorbia heterophylla). R. Bras. Agroc., v. 5, n. 1, p. 22-26, 1999

VIDAL, R. A.; TREZZI, M. M. Análise de crescimento de biótipos de leitera (Euphorbia heterophylla) resistentes e suscetível aos herbicidas inibidores da ALS. Planta Daninha, v. 18, n. 3 , p. $427-433,2000$.

VIDAL, R. A. et al. Definindo resistência aos herbicidas. R. Plan. Dir., v. 2007, p. 18-19, 2007 a.

VIDAL, R. A. et al. Glyphosate resistant biotypes of wild poinsettia (Euphorbia heterophylla L.) and its risk analysis on glyphosate-tolerant soybeans. Int. J. Food Agric. Environ., v. 5, p. 265-269, 2007b 